\title{
Robust Super-Exponential Methods for Blind Deconvolution of MIMO-IIR Systems with Gaussian Noise
}

\author{
Kiyotaka Kohno*, Yujiro Inouye ${ }^{\dagger}$ and Mitsuru Kawamoto ${ }^{\ddagger}$ \\ ${ }^{*}$ Department of Electronic and Control Systems Engineering, Shimane University \\ 1060 Nishikawatsu, Matsue, Shimane 690-8504, Japan \\ ${ }^{\ddagger}$ Information Technology Research Institute, \\ National Institute of Advanced Industrial Science and Technology, \\ Central 2, 1-1-1 Umezono, Tsukuba, Ibaraki 305-8568, Japan \\ *kohno@yonago-k.ac.jp, ${ }^{\dagger}$ inouye@ riko.shimane-u.ac.jp, ${ }^{\ddagger}$ m.kawamoto@ aist.go.jp
}

\begin{abstract}
The so called "super-exponential" methods (SEMs) are attractive methods for solving multichannel blind deconvolution problem. The conventional SEMs, however, have such a drawback that they are very sensitive to Gaussian noise. To overcome this drawback, the robust super-exponential method (RSEM) were proposed for single-input singleoutput infinite impulse response (SISO-IIR) channels and for multi-input multi-output (MIMO) static channels (instantaneous mixtures). While the conventional SEMs use the second- and higher-order cumulants of observations, the RSEM uses only the higher-order cumulants of observations. Since higher-order cumulants are insensitive to Gaussian noise, the RSEM is robust to Gaussian noise. We proposed an RSEM extended to the case of MIMO-IIR channels (convolutive mixtures). To show the validity of the proposed RSEM, some simulation results are presented.
\end{abstract}

\section{INTRODUCTION}

The present paper deals with the multichannel blind deconvolution problem of infinite-impulse response (IIR) channels. To solve this problem, the ideas of the super-exponential methods (SEMs) in [1][3] are used. Several researchers (e.g., [1]-[3]) have so far proposed some SEMs for solving independent component analysis (ICA), blind source separation (BSS) and blind channel equalization (BCE). One of the attractive properties of the SEMs is that the SEMs are computationally efficient and converge to a desired solution at a super-exponential rate. However, almost all the conventional SEMs have such a drawback that they are very sensitive to Gaussian noise, because the conventional SEMs utilize the second-order and the higher-order cumulants of observations.

To overcomes the drawback, Kawamoto et al. proposed new SEMs for SISO-IIR channels [7] and for MIMO static channels (instantaneous mixtures) [6]. The proposed SEMs utilize only the higher-order cumulants of observations, and hence the proposed SEMs become robust to Gaussian noise, so that the proposed SEMs are referred to as robust super-exponential methods (RSEMs).

The purpose of the present paper is to extend the previous idea of RSEMs to the case of MIMO-IIR channels (convolutive mixtures) in the presence of Gaussian noise and to propose an RSEM to this case. One may extend directly the idea to the case of MIMOIIR channels when all the source signals are sub-gaussian or supergaussian. However, this is not the case in general. The extension of the idea is not straightforward in the case when the source signals are of different types, i.e., the sub-gaussian type and the super-gaussian type. It is in need of an assumption on the deconvolvers, which is specified later in this paper. Simulation results are presented to show the effectiveness of the proposed RSEM.

The present paper uses the following notation: Let $Z$ denote the set of all integers. Let $\boldsymbol{C}^{m \times n}$ denote the set of all $m \times n$ matrices with complex components. The superscripts $T, *, H$ and $\dagger$ denote, respectively, the transpose, the complex conjugate, the complex conjugate transpose (Hermitian) and the (Moore-Penrose) pseudoinverse operations of a matrix or a linear operator. The symbols Ker $\boldsymbol{A}$ and $\operatorname{Im} \boldsymbol{A}$ denote the kernel and the image of matrix $\boldsymbol{A}$, respectively. The superscript $\perp$ denotes the orthogonal complement of a subspace. Let $i=\overline{1, n}$ stands for $i=1,2, \cdots, n$.

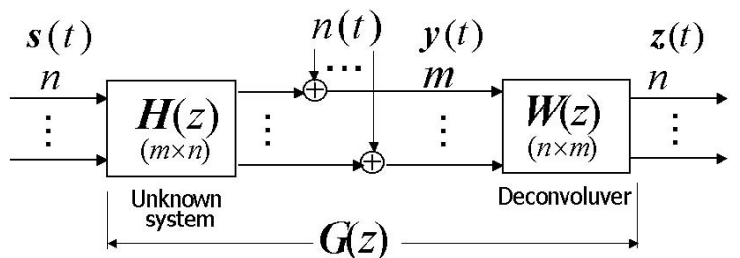

Fig. 1. The composite system of an unknown system and a deconvolver.

\section{PRoblem Formulation}

We consider an MIMO channel with $n$ inputs and $m$ outputs as described by

$$
\boldsymbol{y}(t)=\sum_{k=-\infty}^{\infty} \boldsymbol{H}^{(k)} \boldsymbol{s}(t-k)+\boldsymbol{n}(t), \quad t \in Z,
$$

where $s(t)$ is an $n$-column vector of input (or source) signals, $\boldsymbol{y}(t)$ is an $m$-column vector of channel outputs, $\boldsymbol{n}(t)$ is an $m$-column vector of Gaussian noises, and $\left\{\boldsymbol{H}^{(k)}\right\}$ is an $m \times n$ impulse response matrix sequence.

The transfer function of the channel is defined by

$$
\boldsymbol{H}(z)=\sum_{k=-\infty}^{\infty} \boldsymbol{H}^{(k)} z^{k}, \quad z \in C .
$$

To recover the source signals, we process the output signals by an $n \times m$ deconvolver (or equalizer) $\boldsymbol{W}(z)$ described by

$$
\begin{aligned}
\boldsymbol{z}(\boldsymbol{t}) & =\sum_{k=-\infty}^{\infty} \boldsymbol{W}^{(k)} \boldsymbol{y}(t-k) \\
& =\sum_{k=-\infty}^{\infty} \boldsymbol{G}^{(k)} \boldsymbol{s}(t-k)+\sum_{k=-\infty}^{\infty} \boldsymbol{W}^{(k)} \boldsymbol{n}(t-k),
\end{aligned}
$$

where $\left\{\boldsymbol{G}^{(k)}\right\}$ is the impulse response of the cascade system of the unknown system $\boldsymbol{H}(z)$ and the deconvolver $\boldsymbol{W}(z)$ defined by

$$
\boldsymbol{G}^{(k)}:=\sum_{\tau=-\infty}^{\infty} \boldsymbol{W}^{(\tau)} \boldsymbol{H}^{(k-\tau)}, \quad k \in Z .
$$

The objective of multichannel blind deconvolution is to construct a deconvolver that recovers the original source signals only from the measurements of the corresponding outputs.

We put the following assumptions on the channel and the source signals.

A1) The transfer function $\boldsymbol{H}(z)$ is stable and has full column rank on the unit circle $|z|=1$ [ this implies that the unknown system has less inputs than outputs, i.e., $n \leq m$, and there exists a left stable inverse of the unknown system ].

A2) The input sequence $\{\boldsymbol{s}(t)\}$ is a complex, zero-mean and nongaussian random vector process with element processes $\left\{s_{i}(t)\right\}, i=$ $\overline{1, n}$ being mutually independent. Each element process $\left\{s_{i}(t)\right\}$ is an i.i.d. process with a variance $\sigma_{i}^{2} \neq 0$ and a fourth-order cumulant 
$\gamma_{i} \neq 0$. Moreover, each element process $\left\{s_{i}(t)\right\}$ has nonzero $(p+$ $q+1)$ st-order cumlants $\kappa_{i}$ defined as

$$
\kappa_{i}=\operatorname{cum}\{\underbrace{s_{i}(t), \cdots, s_{i}(t)}_{p}, \underbrace{s_{i}^{*}(t), \cdots, s_{i}^{*}(t)}_{q+1}\} \neq 0,
$$

where $p$ and $q$ are nonnegative integers such that $(p+q) \geq 2$.

A3) The deconvolver $\boldsymbol{W}(z)$ is an FIR channel of sufficient length $L$ so that the truncation effect can be ignored.

A4) The noise sequence $\{\boldsymbol{n}(t)\}$ is a zero-mean, gaussian vector stationary process.

A5) The two vector sequences $\{\boldsymbol{n}(t)\}$ and $\{\boldsymbol{s}(t)\}$ are mutually statistically independent.

Under A3, the impulse response $\left\{\boldsymbol{G}^{(k)}\right\}$ of the cascade system given by

$$
\boldsymbol{G}^{(k)}:=\sum_{\tau=L_{1}}^{L_{2}} \boldsymbol{W}^{(\tau)} \boldsymbol{H}^{(k-\tau)}, \quad k \in Z,
$$

where the length $L:=L_{2}-L_{1}+1$ is taken to be sufficiently large. In a vector form, (6) can be written as

$$
\tilde{\boldsymbol{g}}_{i}=\tilde{\boldsymbol{H}} \tilde{\boldsymbol{w}}_{i}, \quad i=\overline{1, n},
$$

where $\tilde{\boldsymbol{g}}_{\boldsymbol{i}}$ is the column vector consisting of the $i$ th output impulse response of the cascade system defined by

$$
\begin{aligned}
\tilde{\boldsymbol{g}}_{i} & :=\left[\boldsymbol{g}_{i 1}^{T}, \boldsymbol{g}_{i 2}^{T}, \cdots, \boldsymbol{g}_{i n}^{T}\right]^{T}, \\
\boldsymbol{g}_{i j} & :=\left[\cdots, g_{i j}(-1), g_{i j}(0), g_{i j}(1), \cdots\right]^{T},
\end{aligned}
$$

where $g_{i j}(k)$ is the $(i, j)$ th element of matrix $\boldsymbol{G}^{(k)}$, and $\tilde{\boldsymbol{w}}_{i}$ is the $m L$-column vector consisting of the tap coefficients (corresponding to the $i$ th output) of the deconvolver defined by

$$
\begin{aligned}
\tilde{\boldsymbol{w}}_{i} & :=\left[\boldsymbol{w}_{i 1}^{T}, \boldsymbol{w}_{i 2}^{T}, \cdots, \boldsymbol{w}_{i m}^{T}\right]^{T} \in \boldsymbol{C}^{m L}, \\
\boldsymbol{w}_{i j} & :=\left[\boldsymbol{w}_{i j}{ }^{\left(L_{1}\right)}, \boldsymbol{w}_{i j}{ }^{\left(L_{1}+1\right)}, \cdots, \boldsymbol{w}_{i j}{ }^{\left(L_{2}\right)}\right]^{T} \in \boldsymbol{C}^{L},
\end{aligned}
$$

where $w_{i j}{ }^{(k)}$ is the $(i, j)$ th element of matrix $\boldsymbol{W}^{(k)}$, and $\tilde{\boldsymbol{H}}$ is the $n \times m$ block matrix defined by

$$
\tilde{\boldsymbol{H}}:=\left[\begin{array}{cccc}
\boldsymbol{H}_{11} & \boldsymbol{H}_{12} & \cdots & \boldsymbol{H}_{1 m} \\
\boldsymbol{H}_{21} & \boldsymbol{H}_{22} & \cdots & \boldsymbol{H}_{2 m} \\
\vdots & \vdots & \vdots & \vdots \\
\boldsymbol{H}_{n 1} & \boldsymbol{H}_{n 2} & \cdots & \boldsymbol{H}_{n m}
\end{array}\right],
$$

whose $(i, j)$ th block element $\boldsymbol{H}_{i j}$ is the matrix (of $L$ columns and possibly infinite number of rows) with the $(l, r)$ th element $\left[\boldsymbol{H}_{i j}\right]_{l r}$ defined by

$$
\begin{aligned}
& {\left[\boldsymbol{H}_{i j}\right]_{l r}:=h_{j i}(l-r),} \\
& \quad l=0, \pm 1, \pm 2, \cdots, \quad r=L_{1}, L_{1}+1, \cdots, L_{2} .
\end{aligned}
$$

In the multichannel blind deconvolution problem, we want to adjust $\tilde{\boldsymbol{w}}_{i}$ 's $(\mathrm{i}=\overline{1, n})$ so that

$$
\left[\tilde{\boldsymbol{g}}_{1}, \cdots, \tilde{\boldsymbol{g}}_{n}\right]=\tilde{\boldsymbol{H}}\left[\tilde{\boldsymbol{w}}_{1}, \cdots, \tilde{\boldsymbol{w}}_{n}\right]=\left[\tilde{\boldsymbol{\delta}}_{1}, \cdots, \tilde{\boldsymbol{\delta}}_{n}\right] \boldsymbol{P},
$$

where $\boldsymbol{P}$ is an $n \times m$ permutation matrix, and $\tilde{\boldsymbol{\delta}}_{i}$ is the $n$-block column vector defined by

$$
\begin{aligned}
\tilde{\boldsymbol{\delta}}_{i} & :=\boldsymbol{\delta}_{i j}:=\left\{\begin{array}{cl}
{\left[\boldsymbol{\delta}_{i 1}^{T}, \boldsymbol{\delta}_{i 2}^{T}, \ldots, \boldsymbol{\delta}_{i n}^{T}\right]^{T},} \\
\hat{\boldsymbol{\delta}}_{i,}, \quad \text { if } i=j, \\
(\cdots, 0,0,0, \cdots)^{T}, & \text { otherwise } .
\end{array}\right.
\end{aligned}
$$

Here, $\hat{\boldsymbol{\delta}}_{i}$ is the column vector (of infinite elements) whose $r$ th element $\hat{\delta}_{i}(r)$ given by

$$
\hat{\delta}_{i}(r)=d_{i} \delta\left(r-k_{i}\right),
$$

where $\delta(t)$ is the Kronecker delta function, $d_{i}$ is a complex number standing for a scale change and a phase shift, and $k_{i}$ is a integer standing for a time shift.

\section{Robust Super-Exponential Methods}

\section{A. Two-step iterative procedure for vector $\tilde{\boldsymbol{g}}_{i}$}

To find solutions in (14), the following two-step iterative procedure with respect to the elements $g_{i j}, j=\overline{1, n}$ of the vector $\tilde{\boldsymbol{g}}_{i}$ is used:

$$
g_{i j}^{[1]}(k)=\frac{\kappa_{j}}{a_{j}(k) \gamma_{j}}\left(g_{i j}(k)\right)^{p}\left(g_{i j}^{*}(k)\right)^{q}, \quad j=\overline{1, n},
$$

$$
g_{i j}^{[2]}(k)=\frac{g_{i j}^{[1]}(k)}{\sqrt{\sigma_{z_{i}}^{2}}}, \quad j=\overline{1, n},
$$

where $g_{i j}(k)$ in the right-hand side of (18) is an element of $\tilde{\boldsymbol{g}}_{i}$ before the iteration, $(\cdot)^{[1]}$ and $(\cdot)^{[2]}$ stand for the results of the first step and the second step per iteration, $p$ and $q$ are nonnegative integers such that $(p+q) \geq 2, a_{j}(k)$ denotes a positive number (in subsection III.B, it will be shown how we choose the values of $a_{j}(k)$ 's), $\gamma_{j}$ denotes the fourth-order cumulant of $s_{j}(t)$, that is, $\gamma_{j}$ is equal to $\kappa_{j}$ in case of $p=2$ and $q=1$, and $\sigma_{z_{i}}^{2}$ denotes the variance of the output signal $z_{i}(t)$. Equation (18) is derived by replacing $\sigma_{j}^{2}$ of (26) in [2] with $a_{j}(k) \gamma_{j}$, where $\sigma_{j}^{2}$ denotes the second-order cumulant of $s_{j}(t)$, and (19) is used to normalize $g_{i j}^{[1]}$ obtained by (18).

Here it should be noted that in the conventional two-step procedures (e.g., [1]-[3]), the denominator of the right-hand side of (18) was set to 1 or the variance of $s_{j}(t)$, whereas we consider the fourthorder cumulant of $s_{j}(t)$, i.e., $\gamma_{j}$.

Let $g_{i j}^{(l)}(k)$ denote the value obtained in the $l$ th cycle of the iterations of two steps (18) and (19). The important fact of the twostep procedure is that the $n$ values $g_{i j}^{(l)}(k)(j=\overline{1, n})$ converge to zero except for only one of the values as the iteration number $l$ approaches infinity, that is, $l \rightarrow \infty$. The magnitude of the remaining one converges to a positive constant. This will be shown in the following theorem.

Theorem 1: Let $g_{i j}^{(0)}(k)$ be an initial value for iterations of two steps (18) and (19) for each $j=\overline{1, n}$ and $k \in Z$. Let $\alpha_{j}(k)$ be non-negative scalar defined as

$$
\alpha_{j}(k)=\left|\frac{\kappa_{j}}{a_{j}(k) \gamma_{j}}\right|^{\frac{1}{p+q-1}} .
$$

Let $j_{i}$ and $k_{i}$ be $\left(j_{i}, k_{i}\right)=\arg \max _{(j, k)} \alpha_{j}(k)\left|g_{i j}^{(0)}(k)\right|$. Suppose the index $j_{i}$ and $k_{i}$ are unique, that is, $\alpha_{j_{i}}\left(k_{i}\right)\left|g_{i j_{i}}^{(0)}\left(k_{i}\right)\right|>$ $\alpha_{j}(k)\left|g_{i j}^{(0)}(k)\right|$ for any other $j$ and $k$. Then as $l \rightarrow \infty$, it follows

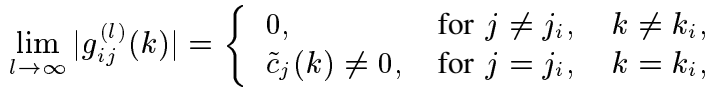

where $\tilde{c}_{j}(k)$ is a scalar positive constant.

Since theorem 1 is proven by using the similar way as in [6], the proof of theorem 1 is omitted for page limit.

For notational simplicity, we confine ourselves to the case $p=$ 2 and $q=1$ (which gives a solution in terms of fourth-order cumulants), although our results are expandable to a general $(p, q)$ case (higher order cumulant case).

We turn to the two-step procedure (18) and (19) with $p=1, q=2$ and $\kappa_{j}=\gamma_{j}(j=\overline{1, n})$. It becomes

$$
\begin{aligned}
& g_{i j}^{[1]}(k)=\frac{1}{a_{j}(k)}\left(g_{i j}(k)\right)^{2}\left(g_{i j}^{*}(k)\right), \quad j=\overline{1, n}, \\
& g_{i j}^{[2]}(k)=\frac{g_{i j}^{[1]}(k)}{\sqrt{\sigma_{z_{i}}^{2}}}, \quad j=\overline{1, n} .
\end{aligned}
$$

\section{B. Two-step iterative procedure for equalizer vector $\tilde{\boldsymbol{w}}_{i}$}

Since the parameters $g_{i j}(k)$ 's involve implicitly the unknown parameters $h_{i j}(k)$ 's, the two-step procedure cannot be handled directly. Therefore, by solving the following weighted least squares problem, we derive an algorithm with respect to $w_{i j}$ 's so that the two steps (22) and (23) can be handled directly.

$$
\min _{\tilde{\boldsymbol{w}}_{i}}\left(\tilde{\boldsymbol{H}} \tilde{\boldsymbol{w}}_{i}-\tilde{\boldsymbol{g}}_{i}\right)^{H} \tilde{\boldsymbol{\Lambda}}\left(\tilde{\boldsymbol{H}} \tilde{\boldsymbol{w}}_{i}-\tilde{\boldsymbol{g}}_{i}\right), \quad i=\overline{1, n} .
$$

Here, $\tilde{\boldsymbol{\Lambda}}$ is a diagonal matrix with positive diagonal elements. The solutions are known to be given by

$$
\tilde{\boldsymbol{w}}_{i}=\left(\tilde{\boldsymbol{H}}^{H} \tilde{\boldsymbol{\Lambda}} \tilde{\boldsymbol{H}}\right)^{\dagger} \tilde{\boldsymbol{H}}^{H} \tilde{\boldsymbol{\Lambda}} \tilde{\boldsymbol{g}}_{i}, \quad i=\overline{1, n} .
$$

In the conventional methods [1]-[3], the positive diagonal elements of $\tilde{\Lambda}$ are set to 1 or the variances of the source signals. This means that $\tilde{\boldsymbol{H}}^{H} \tilde{\boldsymbol{\Lambda}} \tilde{\boldsymbol{H}}$ is calculated by the second-order statistics of the observed signal $\boldsymbol{y}(t)$. We are convinced that this is the reason why the conventional methods are sensitive to Gaussian noise.

In what follows, we shall show that the weighted least squares approach in (24) can be applied to a set of fourth-order cumulants of 
the observed signals $y_{i}(t)(i=\overline{1, m})$, if we choose appropriately a diagonal matrix $\tilde{\Lambda}$ in (24). To this end, we introduce fourthorder cumulants matrices of $m$-vector random process $\{\boldsymbol{y}(t)\}$, which constitute a set of $m \times m$ block matrices $\tilde{\boldsymbol{C}}_{\boldsymbol{y}_{i, j, l}^{(4)}}^{(4)}$, whose elements are defined by

$\left[\tilde{\boldsymbol{C}}_{\boldsymbol{y}_{i, j, l}^{(4)}}^{(4)}\right]_{[p, q]_{l_{1} l_{2}}}=\operatorname{cum}\left\{y_{q}\left(t-l_{2}\right), y_{p}^{*}\left(t-l_{1}\right), y_{j}(t-l), y_{i}^{*}(t-l)\right\}$,

$$
p, q, i, j=\overline{1, m}, \quad l_{1}, l_{2}, l=L_{1}, L_{1}+1, \cdots, L_{2},
$$

where $[\cdot]_{[p, q]_{l_{1} l_{2}}}$ denotes the $\left(l_{1}, l_{2}\right)$ th element of the $(p, q)$ th block matrix of the matrix $\tilde{\boldsymbol{C}}_{\boldsymbol{y}_{i, j, l}}^{(4)}$. Then, we consider an $m \times m$ block matrix $\tilde{\boldsymbol{C}}$ expressed by

$$
\tilde{\boldsymbol{C}}=\sum_{i, j=1}^{m} \sum_{l=L_{1}}^{L_{2}} \beta_{i j} \tilde{\boldsymbol{C}}_{\boldsymbol{y}_{i, j, l}^{(4)}}
$$

where $\beta_{i j}$ 's are either 1 or 0 , which represent design parameters. It is shown by a simple calculation that (27) becomes

$$
\tilde{\boldsymbol{C}}=\tilde{\boldsymbol{H}}^{H} \tilde{\boldsymbol{\Sigma}} \tilde{\boldsymbol{H}}
$$

where $\tilde{\boldsymbol{\Sigma}}$ is a diagonal matrix defined by

$$
\begin{aligned}
& \tilde{\boldsymbol{\Sigma}}:=\operatorname{diag}\left\{\boldsymbol{\Sigma}_{1}, \boldsymbol{\Sigma}_{2}, \cdots, \boldsymbol{\Sigma}_{n}\right\} \\
& \boldsymbol{\Sigma}_{r}:=\operatorname{diag}\left\{\cdots, \gamma_{r} \tilde{a}_{r}(-1), \gamma_{r} \tilde{a}_{r}(0), \gamma_{r} \tilde{a}_{r}(1), \cdots\right\}, r=\overline{1, n} \\
& \tilde{a}_{r}(k):=\sum_{i, j=1}^{m} \sum_{l=L_{1}}^{L_{2}} \beta_{i j} h_{i r}(k-l) h_{j r}^{*}(k-l), k \in Z
\end{aligned}
$$

where $\operatorname{diag}\{\cdots\}$ denotes a diagonal matrix with the diagonal elements built from its arguments.

Here we note that the diagonal matrix $\tilde{\boldsymbol{\Sigma}}$ is not positive semidefinite but the diagonal matrix $\tilde{\Lambda}$ defined by

$$
\begin{aligned}
& \tilde{\boldsymbol{\Lambda}}:=\operatorname{diag}\left\{\boldsymbol{\Lambda}_{1}, \boldsymbol{\Lambda}_{2}, \cdots, \boldsymbol{\Lambda}_{n}\right\} \\
& \boldsymbol{\Lambda}_{r}=\operatorname{diag}\left\{\cdots,\left|\gamma_{r} \tilde{a}_{r}(-1)\right|,\left|\gamma_{r} \tilde{a}_{r}(0)\right|,\left|\gamma_{r} \tilde{a}_{r}(1)\right|, \cdots\right\}
\end{aligned}
$$

is positive semi-definite. It is clear from the definitions (29) and (32) that there exists a sign matrix $\dot{\boldsymbol{I}}$ such that $\tilde{\boldsymbol{\Lambda}}=\tilde{\boldsymbol{\Sigma}} \dot{\boldsymbol{I}}$, where the sign matrix $\dot{\boldsymbol{I}}$ is defined as a diagonal matrix whose diagonal elements are either +1 or -1 .

In (27), let $\beta_{i j}=1$ for $i=j$ and $\beta_{i j}=0$ for $i \neq j$, then $\tilde{a}_{r}(k)$ 's of the diagonal elements of $\tilde{\boldsymbol{\Sigma}}$ become

$$
\tilde{a}_{r}(k)=\sum_{i=1}^{m} \sum_{l=L_{1}}^{L_{2}}\left|h_{i r}(k-l)\right|^{2} \geq 0, \quad r=\overline{1, n}, \quad k \in Z .
$$

Therefore, all the diagonal elements of $\tilde{\boldsymbol{\Sigma}}$ and $\tilde{\boldsymbol{\Lambda}}$ are nonzero except for pathological cases. To avoid completely such pathological cases, the parameters $L_{1}$ and $L_{2}$ in (6) should be chosen to be enough large negative and positive values (let us say, $-\infty$ and $+\infty$ ), respectively. Then $\tilde{a}_{r}(k)$ in (34) approaches to $\tilde{a}_{r}:=\sum_{i=1}^{m} \sum_{l \in Z}\left|h_{i r}(l)\right|^{2}$, where $\tilde{a}_{r}$ is positive for all $r=\overline{1, n}$ under A1.

Theorem 2: Let

$$
\tilde{\boldsymbol{w}}_{i}(\tilde{\boldsymbol{\Lambda}}):=\left(\tilde{\boldsymbol{H}}^{H} \tilde{\boldsymbol{\Lambda}} \tilde{\boldsymbol{H}}\right)^{\dagger} \tilde{\boldsymbol{H}}^{H} \tilde{\boldsymbol{\Lambda}} \tilde{\boldsymbol{g}}_{i}, \quad i=\overline{1, n},
$$

and

$$
\tilde{\boldsymbol{w}}_{i}(\tilde{\boldsymbol{\Sigma}}):=\left(\tilde{\boldsymbol{H}}^{H} \tilde{\boldsymbol{\Sigma}} \tilde{\boldsymbol{H}}\right)^{\dagger} \tilde{\boldsymbol{H}}^{H} \tilde{\boldsymbol{\Sigma}} \tilde{\boldsymbol{g}}_{i}, \quad i=\overline{1, n},
$$

where $\tilde{\boldsymbol{\Lambda}}$ and $\tilde{\boldsymbol{\Sigma}}$ are diagonal invertible matrices, and $\tilde{\boldsymbol{H}}$ and $\tilde{\boldsymbol{g}}_{i}$ 's are defined by (12) and (8) (along with (9)), respectively. Then

(a) If $\tilde{\boldsymbol{\Lambda}}=\tilde{\boldsymbol{\Sigma}}$ or $\tilde{\boldsymbol{\Lambda}}=-\tilde{\boldsymbol{\Sigma}}$, then

$$
\tilde{\boldsymbol{w}}_{i}(\tilde{\boldsymbol{\Lambda}})=\tilde{\boldsymbol{w}}_{i}(\tilde{\boldsymbol{\Sigma}}), \quad i=\overline{1, n} .
$$

(b) If $\boldsymbol{H}(z)$ satisfies assumption $\mathrm{A} 1$ and the dimension $L$ of vectors $\tilde{\boldsymbol{w}}_{i}(\tilde{\boldsymbol{\Lambda}})$ and $\tilde{\boldsymbol{w}}_{i}(\tilde{\boldsymbol{\Sigma}})$ is infinite (i.e., this corresponds to the case $L=$ $L_{2}-L_{1}+1$ with $L_{2}=+\infty$ and $\left.L_{1}=-\infty\right)$, then

$$
\tilde{\boldsymbol{w}}_{i}(\tilde{\boldsymbol{\Lambda}})=\tilde{\boldsymbol{w}}_{i}(\tilde{\boldsymbol{\Sigma}}), \quad i=\overline{1, n} .
$$

To prove the statement (b) of theorem 2, we have recourse to the following lemma.

Lemma 1: Let $\boldsymbol{A}$ be a bounded linear operator of a Hilbert space $\boldsymbol{X}$ into a Hilbert space $\boldsymbol{Y}$ such that $\operatorname{Im} \boldsymbol{A}$ is closed, and $\boldsymbol{B}$ be a bounded linear operator of the Hilbert space $\boldsymbol{Y}$ into a Hilbert space $\boldsymbol{Z}$ such that $\operatorname{Im} \boldsymbol{B}$ is closed. Then the following facts hold true.

(a) If Ker $\boldsymbol{A}=\{0\}$, then $\boldsymbol{A}^{\dagger} \boldsymbol{A}=\boldsymbol{I}$. (b) If $\operatorname{Im} \boldsymbol{A}=(\operatorname{Ker} \boldsymbol{B})^{\perp}$, then $(\boldsymbol{B} \boldsymbol{A})^{\dagger}=\boldsymbol{A}^{\dagger} \boldsymbol{B}^{\dagger}$.

The proof of Lemma 1 is omitted for page limit.

Remark 1: In the finite dimensional case (i.e., the case when $\boldsymbol{A}$ and $\boldsymbol{B}$ are matrices of finite dimension), Lemma 1 is easily proved from the definition of the pseudoinverse (see [4], Section 12.8). However, in the infinite dimensional case (i.e., the case when $\boldsymbol{A}$ and $\boldsymbol{B}$ are linear operators on Hirbert spaces), it is difficult a little, but is proved using properties of the pseudoinverses of linear operators [5].

Proof of theorem 2: (a) If $\underset{\tilde{\boldsymbol{\Lambda}}}{\tilde{\boldsymbol{\Sigma}}} \tilde{\tilde{\boldsymbol{\Sigma}}}$, then (37) follows immediately from (35) and (36). If $\tilde{\boldsymbol{\Lambda}}=-\tilde{\boldsymbol{\Sigma}}$, then (37) also follows immediately from (35) and (36).

(b) First we shall show

$$
\operatorname{Ker} \tilde{\boldsymbol{H}}^{H}=\{0\}
$$

under the conditions that $\boldsymbol{H}(z)$ satisfies A1 and that $L_{1}=-\infty$ and $L_{2}=+\infty$. We note that $\tilde{\boldsymbol{H}}$ becomes a matrix of infinite number of rows and infinite number of columns. Thus, we consider the set $\boldsymbol{l}_{2}$ of column vectors of infinite elements $x_{i}$ 's defined by

$$
\boldsymbol{l}_{2}:=\left\{\left(\cdots x_{0}, x_{1}, \cdots\right)^{T}: \sum_{i \in Z}\left|x_{i}\right|^{2}<\infty\right\} .
$$

Then the set $\boldsymbol{l}_{2}$ is a Hilbert space, and $\tilde{\boldsymbol{H}}$ can be regarded as a linear operator of $\boldsymbol{l}_{2}$ into $\boldsymbol{l}_{2}$. Under the condition that $\boldsymbol{H}(z)$ is stable (i.e., the impulse response $h_{i j}(t)$ of $\boldsymbol{H}(z)$ satisfies $\sum_{i, j \in Z}\left|h_{i j}(t)\right|<\infty$ ), $\boldsymbol{H}$ is a bounded linear operator with the closed image. Thus we can use Lemma 1 for $\tilde{\boldsymbol{H}}$. Let

$$
\tilde{\boldsymbol{y}}:=\tilde{\boldsymbol{H}}^{T} \tilde{\boldsymbol{s}}, \quad \tilde{\boldsymbol{s}} \in \boldsymbol{l}_{2}
$$

where we use the same notation as in (8) and (9) for the elements of $\tilde{\boldsymbol{y}}$ and $\tilde{\boldsymbol{s}}$, and thus they are defined as

$$
\begin{aligned}
\tilde{\boldsymbol{y}} & :=\left[\boldsymbol{y}_{1}^{T}, \boldsymbol{y}_{2}^{T}, \cdots, \boldsymbol{y}_{m}^{T}\right]^{T}, \\
\boldsymbol{y}_{i} & :=\left[\cdots, y_{i}(-1), y_{i}(0), y_{i}(1), \cdots\right]^{T}, i=\overline{1, m} \\
\tilde{\boldsymbol{s}} & :=\left[\boldsymbol{s}_{1}^{T}, \boldsymbol{s}_{2}^{T}, \cdots, \boldsymbol{s}_{n}^{T}\right]^{T}, \\
\boldsymbol{s}_{i} & :=\left[\cdots, s_{i}(-1), s_{i}(0), s_{i}(1), \cdots\right]^{T}, i=\overline{1, n} .
\end{aligned}
$$

In the time domain, (41) is equivalent to

$$
\boldsymbol{y}(t)=\sum_{k=-\infty}^{\infty} \boldsymbol{H}(k) \boldsymbol{s}(t-k), \quad t \in Z
$$

Therefore, suppose $\tilde{\boldsymbol{y}}=0$ which is equivalent to $\boldsymbol{y}(t)=0$ for all $t \in Z$. If follows from (46) and A1 that $s(t)=0$ for all $t \in Z$, which is equivalent to $\tilde{\boldsymbol{s}}=0$. Taking the complex conjugates of elements on the both side of (41), we obtain

$$
\operatorname{Ker} \tilde{\boldsymbol{H}}^{H}=\{0\} \text {. }
$$

On the other hand, it is well known (see [5])

$$
\operatorname{Ker} \tilde{\boldsymbol{H}}^{H}=(\operatorname{Im} \tilde{\boldsymbol{H}})^{\perp} \text {. }
$$

Since $\tilde{\boldsymbol{\Lambda}}$ and $\tilde{\boldsymbol{\Sigma}}$ are invertible, it follows from (47)

$$
\operatorname{Ker} \tilde{\boldsymbol{H}}^{H} \tilde{\boldsymbol{\Lambda}}=\{0\} \quad \text { and } \operatorname{Ker} \tilde{\boldsymbol{H}}^{H} \tilde{\boldsymbol{\Sigma}}=\{0\} .
$$

Using the statement (b) in Lemma 1, (35) becomes

$$
\tilde{\boldsymbol{w}}_{i}(\tilde{\boldsymbol{\Lambda}})=\tilde{\boldsymbol{H}}^{\dagger}\left(\tilde{\boldsymbol{H}}^{H} \tilde{\boldsymbol{\Lambda}}\right)^{\dagger} \tilde{\boldsymbol{H}}^{H} \tilde{\boldsymbol{\Lambda}} \tilde{\boldsymbol{g}}_{i}, \quad i=\overline{1, n},
$$

and (36) becomes

$$
\tilde{\boldsymbol{w}}_{i}(\tilde{\boldsymbol{\Sigma}})=\tilde{\boldsymbol{H}}^{\dagger}\left(\tilde{\boldsymbol{H}}^{H} \tilde{\boldsymbol{\Sigma}}\right)^{\dagger} \tilde{\boldsymbol{H}}^{H} \tilde{\boldsymbol{\Sigma}} \tilde{\boldsymbol{g}}_{i}, \quad i=\overline{1, n} .
$$

Using the statement (a) in Lemma 1, (50) becomes

and (51) becomes

$$
\tilde{\boldsymbol{w}}_{i}(\tilde{\boldsymbol{\Lambda}})=\tilde{\boldsymbol{H}}^{\dagger} \tilde{\boldsymbol{g}}_{i}, \quad i=\overline{1, n}
$$

Therefore, we obtain equalities

$$
\tilde{\boldsymbol{w}}_{i}(\tilde{\boldsymbol{\Sigma}})=\tilde{\boldsymbol{H}}^{\dagger} \tilde{\boldsymbol{g}}_{i}, \quad i=\overline{1, n}
$$

This completes the proof of theorem 2 .

Remark 2: Based on the statement (b) in theorem 2, if the parameters $L_{1}$ and $L_{2}$ of the deconvolver in (11) are chosen to be respectively enough large negative and positive values (let us say, $L_{1} \simeq-\infty$ and $\left.L_{2} \simeq+\infty\right)$, then we have approximate relations

$$
\tilde{\boldsymbol{w}}_{i}(\tilde{\boldsymbol{\Lambda}}) \simeq \tilde{\boldsymbol{w}}_{i}(\tilde{\boldsymbol{\Sigma}}), \quad i=\overline{1, n} .
$$

Therefore, the proposed method can be applied to the case when the 


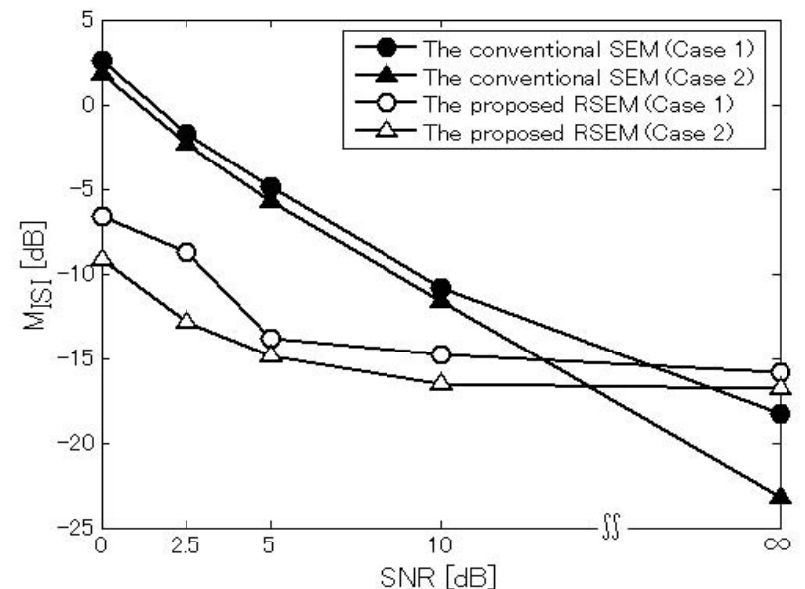

Fig. 2. The performances for the proposed RSEM and the conventional SEM.

signs of the fourth-order cumulants $\gamma_{i}(i=\overline{1, n})$ are different (let us say, we can treat sub-gaussian and super-gaussian signals as the elements of source vector $s(t)$ ).

Moreover, if the MIMO system $\boldsymbol{H}(z)$ is an causal and equalizable FIR system, then there exists an minimum order $L_{0}(\boldsymbol{H})$ of its deconvolvers which attains perfect equalization in the noiseless case. Then it may be conjectured that equalities in (54) hold true if $L \geq L_{0}(\boldsymbol{H})$. However, this is outside the scope of the paper.

For the time being, in the present paper, we consider (27) with $\beta_{i j}$ $=1$ for $i=j$ and $\beta_{i j}=0$ for $i \neq j$. As for $\tilde{\boldsymbol{H}}^{H} \tilde{\boldsymbol{\Sigma}} \tilde{\boldsymbol{H}}$, (27) can be estimated recursively by the fourth-order cumulants block matrices of $\boldsymbol{y}(t)$ using moving averages as shown in [7].

Moreover, as for $\tilde{\boldsymbol{H}}^{H} \tilde{\boldsymbol{\Sigma}} \tilde{\boldsymbol{g}}_{i}$, by using (22) with $a_{j}(k)=\tilde{a}_{j}(k)$ in (34) and the similar way as in [2], it can be calculated by

$$
\begin{aligned}
\tilde{\boldsymbol{D}}_{i} & :=\left[\boldsymbol{d}_{i 1}^{T}, \boldsymbol{d}_{i 2}^{T}, \cdots, \boldsymbol{d}_{i m}^{T}\right]^{T}, \\
{\left[\boldsymbol{d}_{i j}\right]_{l} } & :=\quad \operatorname{cum}\left\{z_{i}(t), z_{i}(t), z_{i}^{*}(t), y_{j}^{*}(t-l)\right\}, \\
i & =\overline{1, n}, \quad j=\overline{1, m}, \quad l=L_{1}, L_{1}+1, \cdots, L_{2} .
\end{aligned}
$$

Then (25) can be expressed as

$$
\tilde{\boldsymbol{w}}_{i}^{[1]}:=\tilde{\boldsymbol{C}}^{\dagger} \tilde{\boldsymbol{D}}_{i}, \quad i=\overline{1, n}
$$

Since the second step (23) is a normalization of $\tilde{\boldsymbol{g}}_{i}$, it is easily shown that the second step reduces to

$$
\tilde{\boldsymbol{w}}_{i}^{[2]}=\tilde{\boldsymbol{w}}_{i}^{[1]} / \sqrt{\sigma_{z_{i}}^{2}} \quad l=\overline{1, n} .
$$

Therefore, (58) and (59) are our proposed two steps to modify $\tilde{\boldsymbol{w}}_{i}$, which constitutes one cycle of iterations in the super-exponential method [1]-[3]. Then since the right-hand side of (58) consists of only fourth-order cumulants, the modification of $\tilde{\boldsymbol{w}}_{i}$ is not affected by Gaussian noise. This comes from the fact that higher-order cumulants are insensitive to additive (even colored) Gaussian noise. This is a novel key point of our proposed super-exponential method, from which the proposed method is referred also to as a robust superexponential method (RSEM).

\section{Simulation Results}

To demonstrate the validity of the proposed RSEM, many computer simulations were conducted. The deflation method [2] was employed for the proposed RSEM in our simulations. Only one of the simulation results is shown in this section. We considered an MIMO channel with two inputs and three outputs, and assumed that the length of channel is three $(K=3)$, that is $\boldsymbol{H}^{(k)} \mathrm{s}$ in (1) were set to be

$$
\begin{aligned}
& \boldsymbol{H}(z)=\sum_{k=0}^{2} \boldsymbol{H}^{(k)} z^{k}= \\
& {\left[\begin{array}{cc}
1.00+0.15 z+0.10 z^{2} & 0.65+0.25 z+0.15 z^{2} \\
0.50-0.10 z+0.20 z^{2} & 1.00+0.25 z+0.10 z^{2} \\
0.60+0.10 z+0.40 z^{2} & 0.10+0.20 z+0.10 z^{2}
\end{array}\right] .}
\end{aligned}
$$

The length of the deconvolver was chosen to be five $(L=5)$. We set the values of the tap coefficients to be zero expect for $w_{11}^{(3)}=w_{22}^{(3)}$ $=1$. Two source signals $s_{1}(t)$ and $s_{2}(t)$ were 4-QAM and 8-QAM signals, respectively. The parameter $p$ and $q$ in (5) were set to be $p$ $=2$ and $q=1$, that is, $\kappa_{j}(j=1,2)$ in (18) were the fourth-order cumulants of the source signals. Three independent Gaussian noises (with identical variance $\sigma_{n}^{2}$ ) were added to the three outputs $y_{i}(t)$ 's at various SNR levels. The SNR is, for convenience, defined as SNR $:=10 \log _{10}\left(\sigma_{s_{i}}^{2} / \sigma_{n}^{2}\right)$, where $\sigma_{s_{i}}^{2}$ 's are the variances of $s_{i}(t)$ 's and are equal to 1 .

As a measure of performance, we used the multichannel intersymbol interference $\left(\mathrm{M}_{\mathrm{ISI}}\right)$ [2],[6]. The value of $\mathrm{M}_{\mathrm{ISI}}$ becomes $-\infty$, if $\tilde{\boldsymbol{g}}_{l}$ 's in (7) are obtained, and hence a minus large value of $\mathrm{M}_{\mathrm{ISI}}$ indicates the proximity to the desired solution. As a conventional method, the method (SEM) proposed in [2] was used for comparison.

Fig. 2 shows the results of performances for both the proposed RSEM and the conventional SEM when the SNR levels were respectively taken to be $0[\mathrm{~dB}]\left(\sigma_{n}^{2}=1\right), 2.5[\mathrm{~dB}], 5[\mathrm{~dB}], 10[\mathrm{~dB}]$, and $\infty$ [dB] $\left(\sigma_{n}^{2}=0\right)$, in which each $\mathrm{M}_{\mathrm{ISI}}$ shown in Fig. 2 was the average of the performance results obtained by 5 independent Monte Carlo runs. In each Monte Carlo run, $\tilde{\boldsymbol{C}}$ and $\tilde{\boldsymbol{D}}_{i}$ were estimated by data samples in the following two cases; (Case 1) 10,000 data and (Case 2) 30,000 data.

It can be seen from Fig. 2 that the proposed RSEM shows better performance than the conventional SEM at SNR less than $10 \mathrm{~dB}$, and as the number of data samples which are needed to estimate the cumulants increases, the proposed RSEM shows much better performance. ,

This implies that the performance of the RSEM depends on the accuracy of the estimates of the higher-order cumulants. We conclude, however, that since in the above two cases, the performances of the RSEM are better than the ones of the conventional SEM for noisy cases, the proposed RSEM is effective for solving the multichannel blind deconvolution problem.

\section{Conclusions}

We proposed an RSEM for deconvolving blindly MIMO-IIR channels in the presence of Gaussian noise. It can treat such general cases as some of the source signals are sub-gaussian and the remainder are super-gaussian. It was shown from the simulation results that the proposed RSEM is robust to Gaussian noise and can successfully solve the multichannel blind deconvolution problem.

\section{ACKNOWLEDGMENT}

The authors would like to thank Shuhei Kobayashi for his cooperation in the computer simulations in the present paper.

\section{REFERENCES}

[1] O. Shalvi and E. Weinstein, "Super-exponential methods for blind deconvolution," IEEE Trans. In formation Theory, vol. 39, no. 2, pp. 504-519, Mar. 1993.

[2] Y. Inouye and K. Tanebe, "Super-exponential algorithms for multichannel blind deconvolution," IEEE Trans. Signal Processing, vol. 48, no. 3, pp. 881-888, Mar. 2000.

[3] M. Martone, "An adaptive algorithm for antenna array low-rank processing in cellular TDMA base stations," IEEE Trans. Commum., vol. 46, no. 5, pp. 627-643, May 1998.

[4] P. Lancaster and M. Tismenetsky, The Theory of Matrices, second edition, Academic Press, INC., 1985.

[5] C. A. Desorer and B. H. Whalen, "A note on pseudoinverses," J. Soc. Indust. Appl. Math., vol. 11, no. 2, pp. 442-447, Jun. 1963.

[6] M. Kawamoto, K. Kohno and Y. Inouye, "Robust Super-Exponential Methods for Deflationary Blind Source Separation of Instantaneous Mixtures," IEEE Trans. Signal Processing, vol. 53, no. 5, pp. 1933-1937, May 2005.

[7] M. Kawamoto, M. Ohata, K. Kohno, Y. Inouye and Asoke K. Nandi, "Robust Super-Exponential Methods for Blind Equalization in The Presence of Gaussian Noise," IEEE Trans. Circuits and Systems-II, vol. 52, no. 10, pp. 651-655, Oct. 2005. 